\title{
Trombocitopenia como preditor de gravidade na dengue em pacientes maiores de 15 anos
}

\author{
Thrombocytopenia as a predictor of severity in dengue in patients older than 15 years \\ Trombocitopenia como predictor de gravedad en el dengue en pacientes mayores \\ de 15 años
}

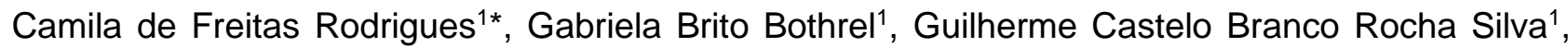
Júlia da Silva Costa ${ }^{1}$, Kathleen Emerick Paiva Faria ${ }^{1}$, Melissa Andrade Meira1, Rafael Alves Macedo ${ }^{1}$, Alexandre Sampaio Moura ${ }^{1}$, Maria Aparecida Turci ${ }^{1}$.

\section{RESUMO}

Objetivo: Verificar se a trombocitopenia tem caráter preditor de gravidade na dengue em pacientes maiores de 15 anos. Métodos: Trata-se de uma revisão sistemática da literatura realizada em fevereiro de 2021 , utilizando o portal da BVS Saúde. Resultados: A busca inicial resultou em 260 artigos, sendo que após a aplicação dos critérios de inclusão e exclusão foram analisados 17 artigos cuja população era composta por adultos maiores de 15 anos de ambos os sexos com diagnóstico confirmado de dengue. O número de pacientes avaliados entre os estudos variou de 32 a 22.777 (mediana: 320). A contagem de plaquetas variou entre as fases agudas e críticas da doença, mantendo-se por volta de $100.000 / \mathrm{mm}^{3}$ na fase aguda, mas atingindo valores de $51.000 / \mathrm{mm}^{3}$ na fase crítica. Cinco estudos avaliaram diferentes valores discriminatórios de plaquetas que se mostraram significativamente associados a desfechos desfavoráveis como óbito e hospitalização prolongada. Esses valores, apesar de variáveis, foram em sua maioria menores ou iguais a $50.000 / \mathrm{mm}^{3}$. Considerações finais: A trombocitopenia foi uma alteração precoce e associada a desfechos desfavoráveis, reforçando a necessidade de manter a contagem de plaquetas na estratificação de gravidade da dengue, podendo atuar inclusive como seu discriminador independente.

Palavras-chave: Trombocitopenia, Dengue, Gravidade, Contagem de plaquetas.

\section{ABSTRACT}

Objective: To verify whether thrombocytopenia is a predictor of severity in dengue in patients older than 15 years. Methods: This is a systematic review of the literature, which was carried out in February 2021 by using the VHL Health portal. Results: The initial search resulted in 260 articles, and after applying the inclusion and exclusion criteria, 17 articles were analyzed whose population was composed of adults over 15 years of both sexes with a confirmed diagnosis of dengue. The number of evaluated patients between the studies ranged from 32 to 22,777 (median: 320 ). The platelet count varied between the acute and critical phases of the disease, remaining around $100,000 / \mathrm{mm}^{3}$ in the acute phase, but reaching values of $51,000 / \mathrm{mm}^{3}$ in the critical one. Five studies evaluated different discriminatory platelet values that were significantly associated with unfavorable outcomes such as death and prolonged hospitalization. Although these values are variable, they were mostly less than or equal to $50,000 / \mathrm{mm}^{3}$. Final considerations: Thrombocytopenia was an early alteration and associated with unfavorable outcomes, which reinforces the need to maintain the platelet count in the stratification of dengue severity, and may even act as its independent discriminator.

Keywords: Thrombocytopenia, Dengue, Severity, Platelet count.

\section{RESUMEN}

Objetivo: Verificar si la trombocitopenia es un predictor de gravedad en el dengue en pacientes mayores de 15 años. Métodos: Se trata de una revisión sistemática de la literatura realizada en febrero de 2021, utilizando el portal BVS Salud. Resultados: La búsqueda inicial resultó en 260 artículos, y después de aplicar los criterios de inclusión y exclusión, 17 artículos cuya población estaba compuesta por adultos mayores de 15 años de ambos sexos con diagnóstico confirmado de dengue. El número de pacientes evaluados entre los estudios osciló entre 32 y 22.777 (mediana: 320). El recuento de plaquetas varió entre las fases aguda y crítica de la enfermedad, permaneciendo alrededor de $100.000 / \mathrm{mm}^{3}$ en la fase aguda, pero alcanzando valores de $51.000 / \mathrm{mm}^{3}$ en la fase crítica. Cinco estudios evaluaron diferentes valores de plaquetas discriminatorios que demostraron estar significativamente asociados con resultados desfavorables como la muerte y la hospitalización prolongada. Estos valores, aunque variables, fueron en su mayoría menores o iguales a $50.000 / \mathrm{mm}^{3}$. Consideraciones finales: La trombocitopenia fue un cambio temprano y se asoció con resultados desfavorables, lo que refuerza la necesidad de mantener el recuento de plaquetas en la estratificación de la gravedad del dengue y puede incluso actuar como su discriminador independiente.

Palabras clave: Trombocitopenia, Dengue, Gravedad, Recuento de plaquetas.

${ }^{1}$ Universidade José do Rosário Vellano (UNIFENAS), Belo Horizonte - MG. *E-mail: camila_freitas11@hotmail.com 


\section{INTRODUÇÃO}

A dengue é uma arbovirose reemergente e endêmica em regiões tropicais e subtropicais localizadas principalmente na África, Américas, Sudeste Asiático e Pacífico Ocidental, sendo esses dois últimos responsáveis por $75 \%$ da população em risco de dengue. O vírus da dengue é responsável por cerca de 390 milhões de novos casos ao ano (BADREDDINE S, et al., 2017), sendo que as taxas de letalidade podem alcançar até $15 \%$ nos pacientes com formas graves da doença. No sudeste Asiático, a dengue afeta principalmente as crianças, enquanto nas Américas é observada em todas as faixas etárias (BRASIL, 2009). O Brasil é o país mais afetado nas Américas, sendo responsável por $70 \%$ dos casos notificados no continente e tem a dengue como importante causa de morbimortalidade (BARROSO ILD, et al., 2020).

A Organização Mundial de Saúde (OMS) divide a dengue em três fases clínicas de acordo com os dias de evolução da doença. A primeira fase é chamada fase febril aguda ou virêmica e ocorre nos três primeiros dias. Segue-se com a fase crítica nos dias quatro a seis, momento em que a maioria dos pacientes apresentam evolução para formas graves, uma vez que seu início é marcado pelo vazamento de plasma. É nesse momento que muitos pacientes procuram atendimento clínico e acabam sendo hospitalizados. A partir do sétimo dia inicia-se a fase de recuperação. Entretanto, nem sempre essas fases se sucedem dessa forma, podendo haver sobreposição entre as fases febril e crítica ou até mesmo evolução diretamente para recuperação sem passar pela fase crítica (JAYADAS TTP, et al., 2019).

Essa doença apresenta um amplo espectro de manifestações clínicas e os pacientes podem ter desde infecção inaparente ou sintomas leves como cefaleia, exantema, mialgia, artralgia e febre com curso autolimitado, até mesmo formas graves, com manifestações hemorrágicas. A febre é um sintoma muito presente nos pacientes e em geral surge nos primeiros quatro dias da doença, de forma repentina, apresentando valores médios entre $38^{\circ}$ a $40^{\circ} \mathrm{C}$ e com duração de aproximadamente dez dias. Vale ressaltar que os sintomas clássicos da dengue estão presentes tanto nos pacientes tratados ambulatorialmente quanto nos hospitalizados. Assim, mesmo os pacientes que são considerados com um quadro leve devem ser informados sobre os possíveis sinais de alerta bem como os profissionais de saúde devem estar atentos à evolução do paciente (HUMAYOUN MA, et al., 2010).

A evolução para formas graves ocorre em uma pequena parcela dos pacientes, e está relacionada ao desenvolvimento da Síndrome do Choque Dengue, caracterizada pelo extravasamento de fluidos, podendo cursar com hipotensão grave, falência dos órgãos e óbito (BRASIL, 2009; LAM PK, et al., 2017). Assim, além de um diagnóstico precoce, torna-se necessário determinar parâmetros clínicos e laboratoriais de fácil acesso que possam estar relacionados com a gravidade da doença, uma vez que se detectados precocemente, podem reduzir desfechos desfavoráveis como hospitalização e morte (BRASIL, 2009).

O tratamento, por sua vez, depende da forma de apresentação da doença, quando autolimitada, consiste em tratar os sintomas, manter o repouso e hidratação; enquanto da forma hemorrágica, baseia-se na internação do paciente para correção do volume perdido e da acidose (BRASIL, 2009). Vale ressaltar que a identificação de preditores clínicos e laboratoriais de sangramento espontâneo, como a presença de petéquias e trombocitopenia pode ser útil tanto na prevenção quanto no tratamento precoce da dengue (DIAZ-QUIJANO FA, et al., 2010).

A infecção pelo vírus da dengue pode causar trombocitopenia por diversos mecanismos como a inibição da produção, o aumento do consumo ou ainda por meio de um distúrbio funcional da plaqueta. A produção é afetada a partir da infecção viral das células progenitoras da medula óssea. Já o consumo, este está aumentado por meio da maior destruição plaquetária imuno mediada além da coagulação intravascular disseminada provocada por mecanismos imunes induzidos pela dengue. Há ainda a perda de funcionalidade da plaqueta relacionada a uma resposta inflamatória exacerbada, que propicia a liberação de interleucina-1B gerando aumento da permeabilidade vascular e consequentemente uma rápida diminuição na contagem de plaquetas (KRISHNAMOORTHY S, et al., 2017; RALAPANAWA U, et al., 2018).

A plaquetopenia geralmente ocorre concomitantemente ao aumento do hematócrito indicativo de extravasamento plasmático que é sugestiva de progressão para vazamento de plasma, característico das formas graves da doença (KRISHNAMOORTHY S, et al., 2017; RALAPANAWA U, et al., 2018). Tradicionalmente utiliza-se o valor do hematócrito na predição de casos potencialmente graves de dengue, 
mas a contagem de plaquetas não é levada em consideração na estratificação da gravidade nas principais diretrizes mundiais, incluindo a do Ministério da Saúde do Brasil (BRASIL, 2009; WORLD HEALTH ORGANIZATION, 2009; PAHO, 2016).

Desta forma, apesar da contagem de plaquetas já ter feito parte do critério de definição de formas graves da dengue e de não se questionar a ocorrência de plaquetopenia em casos que evoluem com maior gravidade, o seu uso como um discriminador independente de gravidade em ferramentas de estratificação de risco tem sido debatido. O objetivo deste trabalho foi verificar se a trombocitopenia tem caráter preditor de gravidade na dengue em pacientes maiores de 15 anos.

\section{MÉTODOS}

Foi realizada uma revisão sistemática da literatura, conduzida em fevereiro de 2021, para responder a pergunta $\mathrm{PI}(\mathrm{E}) \mathrm{CO}$ em que $\mathrm{P}$ eram os pacientes maiores que 15 anos com dengue clássica, a exposição era a trombocitopenia e o $\mathrm{O}$, o desfecho negativo medido pelas hospitalizações e mortes por dengue. A busca sistemática foi realizada no portal da Biblioteca Virtual em Saúde (BVS), que agrega diversas bases de dados como a Medical Literature Analysis and Retrieval System Online (MedLine) e Literatura LatinoAmericana e do Caribe em Ciências da Saúde (Lilacs), através dos termos e da sintaxe da busca de alta sensibilidade (Quadro 1).

Quadro 1 - Sintaxe da busca de alta sensibilidade.

\begin{tabular}{|c|c|}
\hline Estratégica PICO & Descritores utilizados \\
\hline P: população & $\begin{array}{c}\text { (( mh:("Dengue")) OR (dengue) OR (dengue classica) OR (Febre Quebra- } \\
\text { Ossos Febre da Dengue) OR (Infecção pelo Vírus da Dengue) OR (Infecção } \\
\text { por Vírus da Dengue) OR (Infecção por Vírus de Dengue) OR } \\
\text { mh:C01.920.500.270\$ OR mh:C01.925.081.270\$ OR } \\
\text { mh:C01.925.782.350.250.214\$ OR mh:C01.925.782.417.214\$ OR } \\
\text { mh:SP4.012.148.144\$) AND }\end{array}$ \\
\hline I(E): intervenção (exposição) & $\begin{array}{l}\text { (( mh:("Trombocitopenia")) OR (Trombocitopenia) OR (Thrombocytopenia) } \\
\text { OR (Trombocitopenia) OR (Trombopenia) OR mh:C15.378.140.855\$ ) AND }\end{array}$ \\
\hline O: desfecho negativo & $\begin{array}{c}\text { ((Dengue Hemorrágica) OR (Febre Hemorrágica Dengue) OR (Febre } \\
\text { Hemorrágica da Dengue) OR (Febre Hemorrágica devida ao Vírus do } \\
\text { Dengue) OR (Febre Hemorrágica pelo Vírus da Dengue) OR (Febre } \\
\text { Hemorrágica pelo Vírus do Dengue) OR (Síndrome de Choque da Dengue) } \\
\text { OR mh:C01.920.500.270.200\$ OR mh:C01.925.081.270.200\$ OR } \\
\text { mh:C01.925.782.350.250.214.200\$ OR mh:C01.925.782.417.214.200\$ OR ( } \\
\text { mh: "Mortalidade") OR (Mortalidade) OR (Mortality) OR (Mortalidad) OR } \\
\text { (Aumento d\$ Mortalidade) OR (Coeficiente de Mortalidade) OR (Fatores } \\
\text { Determinantes de Mortalidade) OR (Fatores de Mortalidade) OR (Letalidade) } \\
\text { OR (Mortalidade Aumentada) OR (Taxa de Fatalidade) OR (Taxa de } \\
\text { Letalidade) OR (Taxa de Mortalidade) OR (Índice de Casos Fatais) OR } \\
\text { (Índice de Fatalidade) OR (Índice de Letalidade) OR (Índice de Mortalidade) } \\
\text { OR (Evolução Fatal) OR mh:E05.318.308.985.550\$ OR } \\
\text { mh:N01.224.935.698\$ OR mh:N06.850.505.400.975.550\$ OR } \\
\text { mh:N06.850.520.308.985.550\$ OR mh:SP3.076.187.173\$ OR } \\
\text { mh:SP4.046.452.713\$ OR mh:SP4.127.413.629.905\$ OR } \\
\text { mh:SP5.001.002.028.004\$ OR mh:SP5.006.052.168.154\$ OR ( mh: } \\
\text { "Hospitalização") OR (Hospitalização) OR (Hospitalization) OR } \\
\text { (Hospitalización) OR (Internação Hospitalar) OR mh:E02.760.400\$ OR } \\
\text { mh:N02.421.585.400\$ OR mh:VS3.003.001.002\$ OR ( mh:("Prognóstico")) } \\
\text { OR (Prognóstico) OR (Prognosis) OR (Pronóstico) OR (Fatores Prognósticos) } \\
\text { OR mh:E01.789 OR mh:SP5.001.002.028\$) AND (( mh:("Adulto")) OR } \\
\text { (Adulto\$) OR (Adult) OR mh:M01.060.116\$ ) }\end{array}$ \\
\hline
\end{tabular}

Fonte: Rodrigues CF, et al., 2021.

Os critérios de inclusão aplicados foram: pacientes do sexo feminino ou masculino com idade superior a 15 anos, portadores de dengue clássica diagnosticada por sorologia positiva, identificação de PCR ou NS1 positivo e que tenham apresentado algum dos seguintes parâmetros clínicos: febre com duração de dois a sete dias, geralmente alta $\left(39^{\circ} \mathrm{C}\right.$ a $\left.40^{\circ} \mathrm{C}\right)$, de início abrupto, associada à cefaleia, adinamia, mialgia, artralgia, dor retroorbitária, anorexia, náuseas, vômitos e exantema. Foram excluídos estudos que utilizassem apenas crianças ou apenas pacientes com dengue grave e estudos que não utilizassem a contagem de plaquetas como preditor de gravidade, além de estudos não encontrados para leitura completa ou que fossem de idioma diferente de português, inglês e espanhol. Além disso, artigos publicados há mais de dez anos também não foram incluídos nesta revisão. 
Os artigos identificados pela busca foram avaliados de forma independente por uma dupla de revisores por meio da plataforma de seleção Rayyan em modo "blind on". Nesse momento foram identificadas e excluídas as duplicatas. O rastreamento dos estudos foi feito pela leitura dos títulos e resumos e os estudos selecionados por pelo menos um dos revisores foram para a próxima etapa. A seleção foi feita pela leitura do texto completo, também de forma independente e em modo "blind on". Nessa etapa, os motivos de exclusão foram registrados e a discordância entre os autores foi discutida em reunião de consenso.

Os dados extraídos foram título, autores, revista, ano de publicação, critérios utilizados para o diagnóstico da dengue, número de pacientes avaliados, idade, sexo, comorbidades, contagem de plaquetas, manifestações clínicas da dengue, morte por dengue grave e complicações. A síntese será apresentar os estudos segundo suas características e principais resultados.

\section{RESULTADOS}

Através da busca de alta sensibilidade foram encontrados 255 artigos e após a exclusão de seis duplicidades restaram 249. Além disso, foram incluídos cinco artigos provenientes de busca manual, resultando em 254. A partir da leitura de títulos e resumos de todos os estudos por uma dupla de revisores, 79 artigos foram pré-selecionados para serem lidos na íntegra. Dos 79 artigos inicialmente triados, após a aplicação de critérios de inclusão e exclusão, 17 foram revisados ao final (Figura 1).

Figura 1 - Fluxograma metodológico para seleção dos artigos.
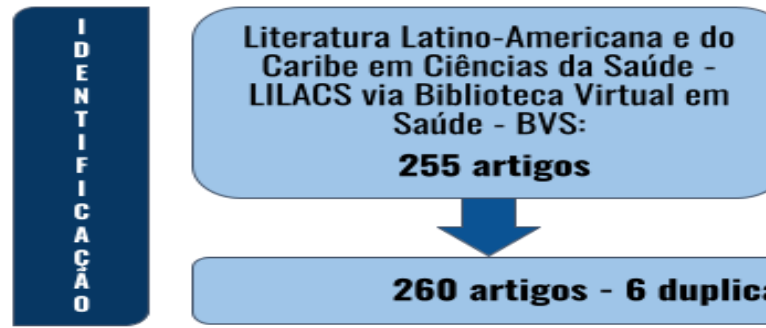

\section{Estudos de outras fontes: 5 artigos}

260 artigos -6 duplicatas $=254$ artigos
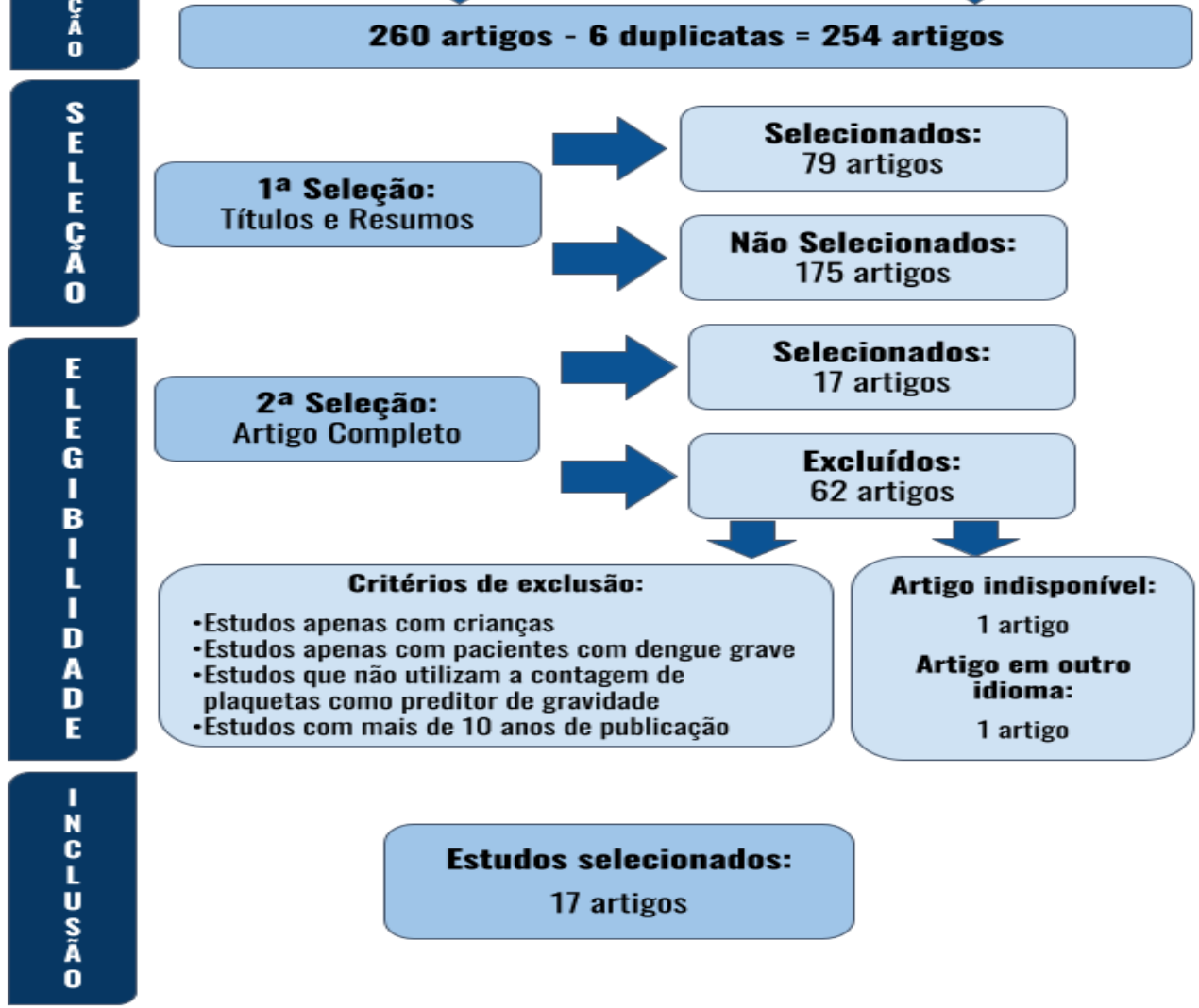

Estudos selecionados: 17 artigos

Fonte: Rodrigues CF, et al., 2021.

Foram incluídos nesta revisão dezessete artigos que contemplaram os critérios de inclusão. O número de pacientes avaliados entre os estudos variou de 32 a 22.777 , com mediana de 320 , incluindo pacientes do sexo masculino e feminino (Quadro 2). 
Quadro 2 - Características dos estudos avaliados.

\begin{tabular}{|c|c|c|c|}
\hline Identificação do estudo Autor (ano) & $\begin{array}{c}\text { Número de pacientes } \\
\text { avaliados }\end{array}$ & $\begin{array}{c}\text { Idade média } \\
\text { (anos) }\end{array}$ & Sexo (masculino) \\
\hline BADREDDINE S, et al. (2017) & 482 & 34 & - \\
\hline CHAMNANCHANUNT S, et al. (2012) & 97 & - & $40,20 \%$ \\
\hline DHANOA A, et al. (2017) & 237 & 27.7 & $56,96 \%$ \\
\hline FERNÁNDEZ E, et al. (2017) & 320 & 22,4 & $56,56 \%$ \\
\hline FLAMAND C, et al. (2017) & 1356 & 25 & $46,31 \%$ \\
\hline HO TS, et al. (2013) & 309 & 48 & $46,92 \%$ \\
\hline HUMAYOUN MA, et al. (2010) & 110 & - & $63,63 \%$ \\
\hline JAYADAS TTP, et al. (2019) & 78 & - & $69,23 \%$ \\
\hline KRISHNAMOORTHY S, et al. (2017) & 1308 & 52 & $54,89 \%$ \\
\hline LEE IK, et al. (2018) & 1086 & 32 & $47,23 \%$ \\
\hline NIRIELLA MA, et al. (2020) & 697 & 32,8 & $51,21 \%$ \\
\hline RALAPANAWA U, et al. (2018) & 515 & 35 & $66,60 \%$ \\
\hline TAVAKOLIPOOR P, et al. (2016) & 119 & - & $56,30 \%$ \\
\hline THOMAS L, et al. (2010) & 560 & - & $46,96 \%$ \\
\hline VISHNURAM P, et al. (2018) & 32 & 36 & $75 \%$ \\
\hline WONG JGX, et al. (2016) & 188 & 45,5 & $42,55 \%$ \\
\hline YEH CY, et al. (2017) & 22.777 & $49,64 \%$ \\
\hline
\end{tabular}

Fonte: Rodrigues CF, et al., 2021.

Sete estudos apresentaram as comorbidades das populações estudadas. As mais frequentemente citadas foram Diabetes Mellitus, Hipertensão Arterial Sistêmica e Doença Renal Crônica (DRC). Nessa revisão 328 pacientes eram diabéticos, 297 eram hipertensos e 16 portadores de DRC. Em relação às manifestações clínicas mais frequentes, a febre esteve presente na maioria dos pacientes (22681), uma vez que essa fez parte da definição diagnóstica dos casos na maior parte dos estudos. Ao contrário, sintomas de sangramento espontâneo, como as petéquias (383) foram os menos observados. Além dessas também foram frequentes mialgia ou artralgia (7630), cefaleia (6505), dor abdominal (1216) e náusea ou vômito (884).

Seis estudos avaliaram o valor médio da contagem de plaquetas em pacientes que evoluíram com dengue grave (Gráfico 1). Outros três estudos também avaliaram a contagem de plaquetas, mas analisaram os dados levando em consideração a porcentagem de pacientes que apresentaram contagem abaixo de determinado limite. Este limite variou entre os artigos, com alguns autores considerando o limite como $150.000 / \mathrm{mm}^{3}$ e outros optando por um limite mais baixo de $50.000 / \mathrm{mm}^{3}$ (Gráfico 2).

Gráfico 1 - Valor médio da contagem de plaquetas em pacientes que evoluíram com dengue grave.

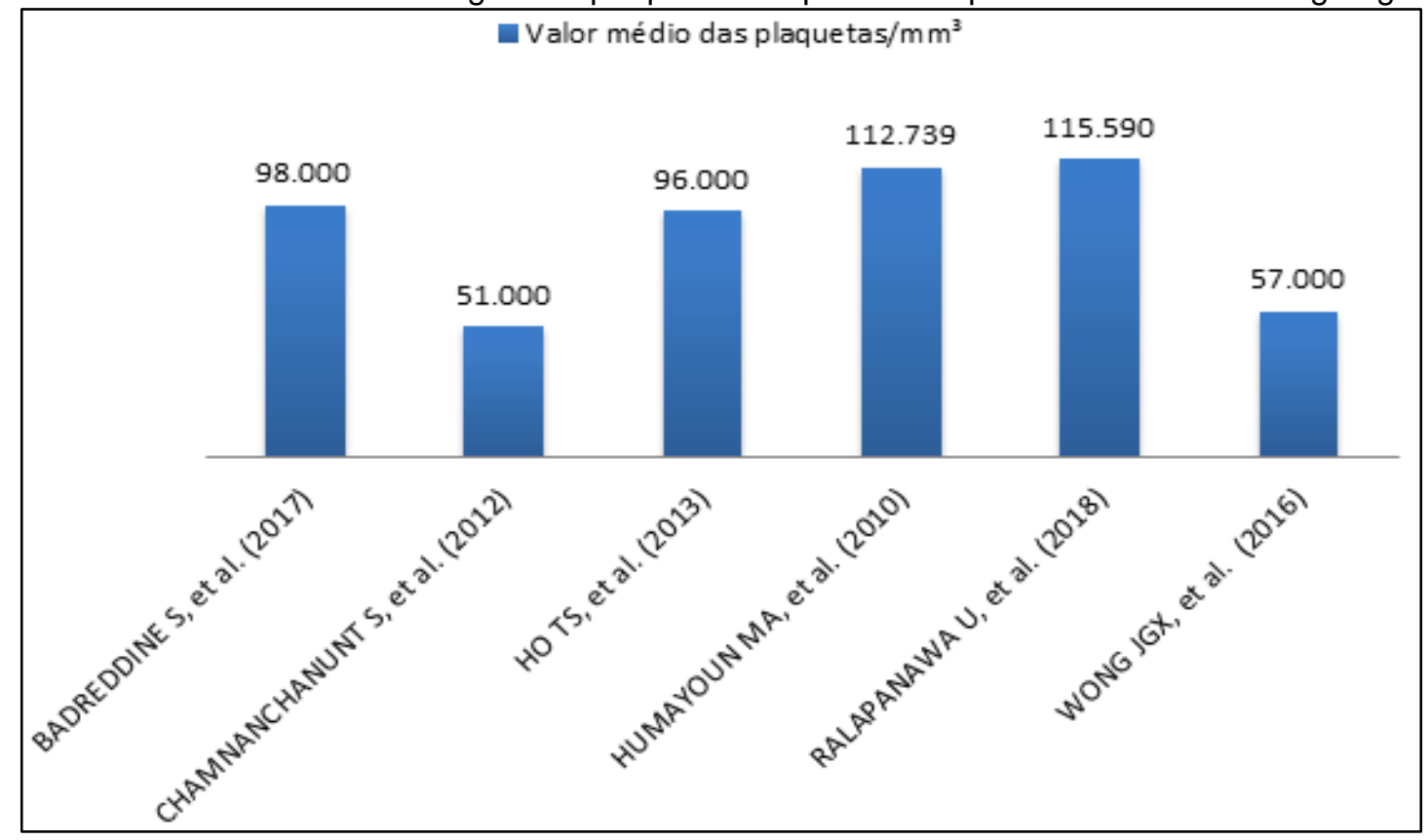

Fonte: Rodrigues CF, et al., 2021. 
Gráfico 2 - Proporção de pacientes com contagem de plaquetas abaixo de determinado limite, em pacientes com dengue grave

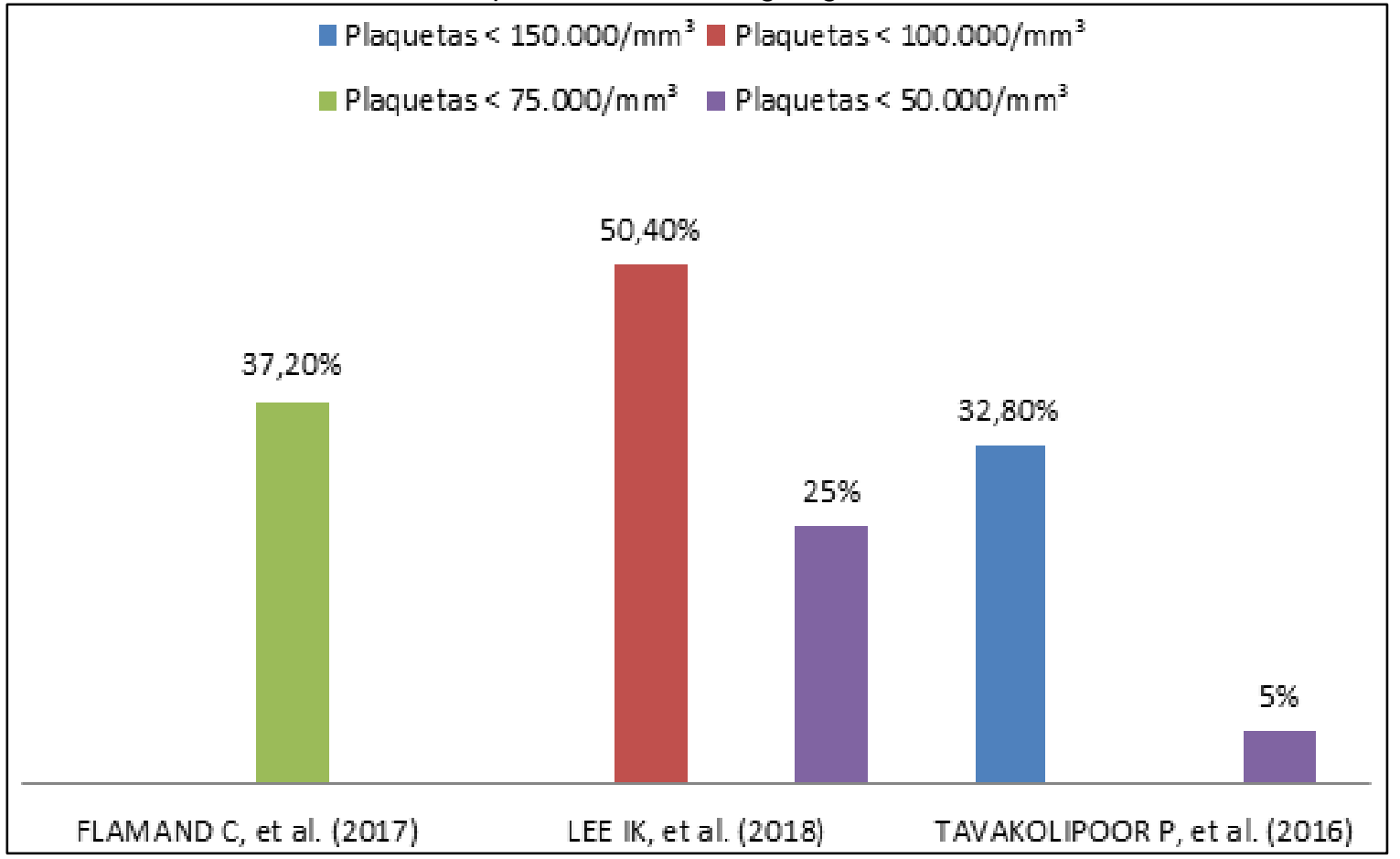

Fonte: Rodrigues CF, et al., 2021.

Dois trabalhos fizeram uma comparação do valor médio das plaquetas entre grupos de pacientes com dengue clássica e aqueles que evoluíram com sangramento clinicamente significativo (Quadro 3).

Quadro 3 - Análise comparativa entre pacientes com sangramento clinicamente significativo e pacientes com sangramento não clinicamente significativo.

\begin{tabular}{|c|c|c|c|c|c|}
\hline \multirow[b]{2}{*}{ Estudo } & \multicolumn{2}{|c|}{ Número de pacientes } & \multicolumn{2}{|c|}{ Contagem de plaquetas (/mm3) } & \multirow[b]{2}{*}{$\begin{array}{l}\text { Valor } \\
\text { de } p\end{array}$} \\
\hline & $\begin{array}{l}\text { Pacientes com } \\
\text { sangramento } \\
\text { significativo }\end{array}$ & $\begin{array}{c}\text { Pacientes sem } \\
\text { sangramento } \\
\text { significativo }\end{array}$ & $\begin{array}{c}\text { Pacientes com } \\
\text { sangramento } \\
\text { significativo }\end{array}$ & $\begin{array}{c}\text { Pacientes sem } \\
\text { sangramento } \\
\text { significativo }\end{array}$ & \\
\hline $\begin{array}{c}\text { CHAMNANCHANUNT S, } \\
\text { et al. (2012) }\end{array}$ & 97 & 180 & 51.000 & 76.000 & 0,007 \\
\hline WONG JGX, et al. (2016) & 188 & 4195 & 57.000 & 63.000 & 0,004 \\
\hline
\end{tabular}

Fonte: Rodrigues CF, et al., 2021.

Os valores da contagem de plaquetas foram diferentes de acordo com as fases clínicas da doença, variando em média de $121.600 / \mathrm{mm}^{3}$ na fase febril aguda e $80.630 / \mathrm{mm}^{3}$ na fase crítica (JAYADAS TTP, et al. 2019). Já o estudo de Ralapanawa U, et al. (2018) variou de $154.360 / \mathrm{mm}^{3}$ na fase aguda e $108.500 / \mathrm{mm}^{3}$ na fase crítica.

Em relação aos desfechos da evolução da dengue, a morte foi avaliada por onze artigos, sendo que em um deles, nenhum paciente evoluiu para óbito. Dentre os 29574 pacientes avaliados nesta revisão, 280 evoluíram para óbito, o que representa $0,9 \%$. As principais complicações avaliadas pelos estudos foram necessidade de hospitalização ( $n=1072)$; internação em Unidades de Cuidados Intensivos $(n=364)$; transfusão de plaquetas $(n=8)$ e evolução com hemorragia $(n=463)$.

Os valores de trombocitopenia associados à evolução com desfecho desfavorável como repercussões graves, hospitalização ou óbitos variaram de $<20.000 / \mathrm{mm}^{3}$ a $<120.000 / \mathrm{mm}^{3}$ (Quadro 4). 
Quadro 4 - Associação entre contagem de plaquetas e desfechos clínicos desfavoráveis em diferentes estudos.

\begin{tabular}{|c|c|c|c|c|c|c|}
\hline Estudo & $\mathbf{n}$ & Desfecho & $\begin{array}{l}\text { Contagem } \\
\text { de } \\
\text { plaquetas } \\
(/ \mathrm{mm} 3)\end{array}$ & $\begin{array}{l}\text { Outras variáveis } \\
\text { incluídas na análise } \\
\text { multivariada }\end{array}$ & $\begin{array}{c}\text { Associação } \\
\text { entre } \\
\text { plaquetopenia } \\
\text { e desfecho } \\
\text { Razão de } \\
\text { chance } \\
\text { ajustada (IC) }\end{array}$ & $\begin{array}{l}\text { Valor } \\
\text { de p }\end{array}$ \\
\hline $\begin{array}{c}\text { DHANOA A, et al. } \\
(2017)\end{array}$ & 237 & $\begin{array}{l}\text { internação } \\
>3 \text { dias }\end{array}$ & $\leq 20.000$ & $\begin{array}{c}\text { Comorbidades (DM, } \\
\text { asma, HAS, doença } \\
\text { cardiovascular, } \\
\text { neoplasia), } \\
\text { hemoconcentração, } \\
\text { dosagem de albumina. }\end{array}$ & $\begin{array}{c}1,943 \\
(1,01-3,77)\end{array}$ & $<0,05$ \\
\hline $\begin{array}{l}\text { FLAMAND C, et } \\
\text { al. }(2017)\end{array}$ & 1356 & $\begin{array}{l}\text { dengue } \\
\text { grave }\end{array}$ & $\leq 75.000$ & $\begin{array}{l}\text { Extravasamento de } \\
\text { plasma, dosagem de } \\
\text { transaminases, } \\
\text { hemoconcentração, } \\
\text { idade > } 16 \text { anos }\end{array}$ & $\begin{array}{c}2,25 \\
(1,60-3,18)\end{array}$ & $<0,001$ \\
\hline $\begin{array}{l}\text { KRISHNAMOORT } \\
\text { HY S, et al. (2017) }\end{array}$ & 1308 & óbito & $<20.000$ & - & $\begin{array}{c}3,59 \\
(1,13-9,77)\end{array}$ & $<0,05$ \\
\hline $\begin{array}{l}\text { LEE IK, et al. } \\
\qquad(2018)\end{array}$ & 1086 & óbito & $<50.000$ & $\begin{array}{c}\text { Sangramento } \leq 72 \mathrm{~h} \\
\text { após apresentação, } \\
\text { leucocitose e } \\
\text { hemoconcentração }\end{array}$ & $\begin{array}{c}5,42 \\
(1,39-21,02)\end{array}$ & 0,015 \\
\hline $\begin{array}{l}\text { NIRIELLA MA, et } \\
\text { al. (2020) }\end{array}$ & 697 & $\begin{array}{l}\text { dengue } \\
\text { grave }\end{array}$ & $<120.000$ & $\begin{array}{c}\text { Idade 41-50 anos, sexo } \\
\text { feminino, DM, vômitos } \\
\text { persistentes, } \\
\text { transaminases }\end{array}$ & $\begin{array}{c}1,88 \\
(1,39-2,64)\end{array}$ & $<0,01$ \\
\hline $\begin{array}{l}\text { YEH CY, et al. } \\
\qquad(2017)\end{array}$ & 22777 & óbito & $<100.000$ & $\begin{array}{l}\text { Idade, dificuldade } \\
\text { respiratória, alteração } \\
\text { da consciência }\end{array}$ & $\begin{array}{c}2,55 \\
(1,24-5,28)\end{array}$ & $<0,0001$ \\
\hline
\end{tabular}

Legenda: IC: Intervalo de Confiança; n: Número de pacientes avaliados; DM: Diabetes Mellitus; HAS: Hipertensão Arterial Sistêmica

Fonte: Rodrigues CF, et al., 2021.

\section{DISCUSSÃO}

Embora a maioria dos casos de dengue tenha evolução autolimitada, uma pequena proporção evolui para formas graves e, ocasionalmente, para o óbito. Essa progressão é multifatorial e envolve interações entre subtipos virais, genética, histórico imunológico do hospedeiro, comorbidades, idade, sexo e parâmetros laboratoriais, como aumento de hematócrito e queda na contagem das plaquetas. Já é estabelecido que infecções prévias por subtipos virais da dengue estão relacionadas à maior gravidade se nova infecção. Logo, locais endêmicos para diferentes subtipos assim como o perfil clínico-epidemiológico de cada população interferem de forma diferente na história natural da doença, o que implica em adequações de recursos para aspectos de prevenção, manejo e cura da doença (FLAMAND C, et al., 2017).

Diante de um paciente com suspeita clínica de dengue uma das etapas mais importantes é a realização da estratificação de risco com identificação dos pacientes com maior chance de evolução clínica desfavorável. Esta estratificação pode ser feita a partir dos sinais de alarme que incluem parâmetros laboratoriais simples e de baixo custo como o hematócrito e plaquetas. Tratam-se de exames amplamente disponíveis na rede pública, privada e suplementar de saúde, podendo ser usados como complemento na avaliação clínica de pacientes potencialmente graves. A diferenciação entre casos leves e graves tem impacto diretamente na gestão de saúde, uma vez que muitos pacientes são internados desnecessariamente. Essa situação onera os gastos em saúde e ainda expõe os pacientes a riscos adicionais durante o período de internação (HO TS, et al., 2013).

No presente estudo foi visto que a trombocitopenia pode funcionar como um preditor de gravidade para dengue uma vez que está correlacionada à evolução para desfechos desfavoráveis. Assim, identificar a contagem de plaquetas precocemente pode otimizar a evolução e o prognóstico do paciente. Os valores da contagem de plaquetas variam conforme a fase da doença sendo mais altos durante a fase febril aguda (dia 
2 e 3) e mais baixos durante a fase crítica (dia 5) da doença, podendo apresentar quedas bruscas no período de transição entre as fases. Já o nadir da contagem de plaquetas foi encontrado entre $\circ 5^{\circ}$ e $\circ 6^{\circ}$ dia de início da febre, o que demonstra a importância de acompanhar a evolução clínica e laboratorial desses pacientes (BADREDDINE S, et al., 2017).

Considerando que os menores valores de contagem de plaquetas comumente são encontrados na fase crítica, ao encontrá-los na fase febril aguda isso demonstra necessidade de acompanhamento rigoroso de tais pacientes, com eventual repetição da contagem de plaquetas, uma vez que nessas situações a chance de evolução com dengue grave torna-se maior. Diante do grande número de pacientes acometidos durante um surto de dengue, é de extrema importância identificar precocemente pacientes que irão evoluir para desfechos desfavoráveis e assim intervir de forma a garantir o acesso ao plano de cuidado necessário (BADREDDINE S, et al., 2017).

Além da trombocitopenia, outras alterações laboratoriais aparecem precocemente, como o aumento do hematócrito. Esse foi avaliado pelos estudos, Tavakolipoor P, et al. (2016), Chamnanchanunt S, et al. (2012), Vishnuram P, et al. (2018), Fernández E, et al. (2017), Lee IK, et al. (2018), Dhanoa A, et al. (2017) e Jayadas TTP, et al. (2019), e reflete um aumento da permeabilidade dos vasos sanguíneos, o que leva ao extravasamento de plasma que pode resultar em choque se não abordado corretamente Dois desses estudos, Fernández E, et al. (2017) e Jayadas TTP, et al. (2019), demonstraram um aumento percentual do hematócrito com a progressão da doença, o que em geral coincide com o momento em que as plaquetas estão mais baixas, demonstrando a relação entre esses dois parâmetros laboratoriais.

As formas graves da dengue dependem de uma interação de fatores como a alteração na permeabilidade vascular, distúrbios da hemostasia e hipotensão. Nessa última instância os pacientes evoluem de forma mais grave com eventual choque hipovolêmico, que pode causar a morte. Um dos sinais de alerta, com grande potencial para evolução com formas graves, incluem os sangramentos, e esses foram avaliados por dois estudos que dividiram seus pacientes em grupos com sangramento clinicamente significativo e sem sangramento clinicamente significativo. Como esperado, a contagem de plaquetas no grupo que apresentou sangramento com repercussão clínica foi mais baixa. Além disso, a chance de sangramento era maior quando os níveis de plaquetas estavam abaixo de $25.000 / \mathrm{mm}^{3}$ (CHAMNANCHANUNT S, et al. 2012; WONG JGX, et al. 2016).

Nesta revisão as petéquias foram manifestações presentes ainda que em pequena parte dos pacientes. No entanto, vale ressaltar que esse é um sinal clínico que aparece mais tardiamente sendo mais frequente em pacientes com dengue grave, demonstrando a ineficácia desse parâmetro para predizer evolução desfavorável. A baixa prevalência desse sinal no presente estudo justifica-se pelo fato de que a população avaliada foi de pacientes em potencial evolução para as formas graves da dengue (KRISHNAMOORTHY S, et al., 2017).

Desde a década de 1970, a dengue era classificada em dengue indiferenciada, dengue clássica, febre hemorrágica da dengue (FHD) e síndrome do choque da dengue. Nesta classificação, a contagem de plaquetas era fundamental, pois valores abaixo de $100.000 / \mathrm{mm}^{3}$ eram um dos critérios essenciais para se definir um caso como FHD. No entanto, em 2008, a partir de um acordo global entre especialistas, foi proposta uma nova classificação composta por apenas três categorias: dengue sem sinais de alarme, dengue com sinais de alarme e dengue grave (BRASIL, 2009, OPS/OMS, 2010; BRASIL, 2013).

$\mathrm{Na}$ nova classificação, para estratificar um quadro como dengue grave não era mais necessária a contagem de plaquetas. Nas linhas de cuidado publicadas pela OMS, Organização Pan-Americana da Saúde (OPAS/PAHO) e Ministério da Saúde após a classificação revisada, as plaquetas ainda faziam parte dos sinais de alarme, mas não como um critério isolado e sim associada à elevação progressiva do hematócrito (BRASIL, 2009, OPS/OMS, 2010; BRASIL, 2013).

Nessas linhas de cuidado atualizadas, a contagem de plaquetas acima de $50.000 / \mathrm{mm}^{3}$ como critério para alta também foi substituída por um critério mais genérico, definido como "plaquetas em ascensão". A PAHO (2016) atualizou suas recomendações e as plaquetas não eram mais listadas como parte dos sinais de alarme, sendo também suprimidas na publicação subsequente das recomendações de manejo clínico da dengue do Ministério da Saúde do Brasil do mesmo ano (PAHO, 2016; BRASIL, 2016). 
A presente revisão mostra que é inadequado não se mencionar as plaquetas como um critério relevante na estratificação de risco dos pacientes com suspeita de dengue tendo em vista que esta se mostrou associada a diferentes desfechos desfavoráveis como morte, hospitalização, necessidade de terapia intensiva, transfusão de plaquetas e presença de sangramentos clinicamente significativos. Um outro desfecho grave e potencialmente fatal de evolução da dengue também caracterizado pela presença de trombocitopenia grave é o desenvolvimento de hepatite com progressão para insuficiência hepática (KRISHNAMOORTHY S, et al., 2017).

Destaca-se que três estudos demonstraram que a associação entre contagem de plaquetas e evolução desfavorável permanece mesmo após análise ajustada para valores de hemoconcentração, ou seja, a contagem de plaquetas constitui-se um marcador de risco de maneira independente do hematócrito (DHANOA A, et al., 2017; FLAMAND C, et al., 2017; LEE IK, et al., 2018).

A principal limitação deste trabalho está na dificuldade em se definir um ponto de corte para a contagem das plaquetas que aumenta o risco de evolução desfavorável em um paciente com dengue. A classificação em trombocitopenia leve, moderada e grave difere entre os estudos. Além disso, alguns estudos utilizam apenas uma medida da contagem de plaquetas realizada em pacientes já hospitalizados, o que compromete a avaliação longitudinal desse parâmetro em uma doença que é bastante dinâmica (WONG JGX, et al., 2016).

Outra limitação do estudo está relacionada a grandes variações do tamanho amostral entre os artigos analisados. $\mathrm{O}$ trabalho de Vishnuram $\mathrm{P}$, et al. (2018) englobou apenas 32 pacientes, enquanto o trabalho de Yeh CY, et al. (2017), teve uma população avaliada de 22.777 pacientes. Dessa forma, considerando que quanto maior a população envolvida na análise, maior a confiabilidade dos achados, os resultados encontrados nos diferentes estudos devem ser analisados levando em consideração o tamanho da amostra e a significância estatística dos valores avaliados. Vale a pena ressaltar que a mediana de participantes nos estudos que avaliaram a associação da contagem de plaquetas com desfecho desfavorável foi elevada, de 1197 pessoas.

Destaca-se ainda que apenas três estudos analisaram pacientes ambulatoriais o que limita a extrapolação dos achados para tomada de decisão em relação ao uso da contagem de plaquetas para indicar a hospitalização de casos em maior risco de agravamento. Os resultados também não podem ser estendidos para crianças tendo em vista que apenas estudos em adultos foram utilizados nesta revisão (FLAMAND C, et al., 2017; TAVAKOLIPOOR P, et al. 2016; BADREDDINE S, et al., 2017).

\section{CONSIDERAÇÕES FINAIS}

Nesta revisão, a trombocitopenia moderada/grave se mostrou como um fator preditor de gravidade em pacientes maiores de 15 anos, estando associada de maneira independente a formas clínicas de maior gravidade, internação e óbito. Apesar das discrepâncias em relação ao ponto de corte do valor de plaquetas, a maior parte dos estudos mostrou que valores iguais ou inferiores a $50.000 / \mathrm{mm}^{3}$ estiveram associados a um pior prognóstico. Os achados desta revisão sistemática apontam para a necessidade da monitorização da contagem de plaquetas ao longo das diferentes fases da dengue e para a manutenção da contagem de plaquetas como um parâmetro na estratificação de gravidade da doença.

\section{REFERÊNCIAS}

1. BADREDDINE S, et al. Dengue fever: Clinical features of 567 consecutive patients admitted to a tertiary care center in Saudi Arabia. Saudi Med J, 2017; 38(10): 1025-1033.

2. BARROSO ILD, et al. Um estudo sobre a prevalência da dengue no Brasil: Análise da literatura. Braz. J. of Develop., 2020; 6(8): 61878-61883.

3. BRASIL. Ministério da Saúde. Secretaria de Vigilância em Saúde. Departamento de Vigilância das Doenças Transmissíveis. Dengue: diagnóstico e manejo clínico : adulto e criança. 2016. Disponível em: https://portalarquivos2.saude.gov.br/images/pdf/2016/janeiro/14/dengue-manejo-adulto-crianca-5d.pdf Acessado em: 05 de fevereiro de 2020 . 
4. BRASIL. Ministério da Saúde. Secretaria de Vigilância em Saúde. Departamento de Vigilância Epidemiológica. Diretrizes nacionais para prevenção e controle de epidemias de dengue. 2009. Disponível em: http://bvsms.saude.gov.br/bvs/publicacoes/diretrizes_nacionais_prevencao_controle_dengue.pdf. Acessado em: 05 de fevereiro de 2021.

5. BRASIL. Ministério da Saúde. Secretaria de Vigilância em Saúde. Diretoria Técnica de Gestão. Dengue: diagnóstico e manejo clínico: adulto e criança. 2013. Disponível em: http://bvsms.saude.gov.br/bvs/publicacoes/dengue_diagnostico_manejo_clinico_adulto.pdf Acessado em: 05 de fevereiro de 2020.

6. CHAMNANCHANUNT $S$, et al. Early predictors of clinically significant bleeding in adults with dengue infection. Southeast Asian J Trop Med Public Health, 2012; 43(4): 890-899.

7. DHANOA A, et al. Risk factors and clinical outcome of profound thrombocytopenia in adult patients with DENV infections. Platelets, 2017; 28(7): 724-727.

8. DIAZ-QUIJANO FA, et al. Predictors of spontaneous bleeding in patients with acute febrile syndrome from a dengue endemic area. J Clin Virol, 2010; 49(1): 11-15.

9. FERNÁNDEZ E, et al. A retrospective cohort study to predict severe dengue in Honduran patients. BMC Infect Dis, 2017; 17(1): 1-6.

10. FLAMAND C, et al. Epidemiological assessment of the severity of dengue epidemics in French Guiana. PLoS One, 2017; 12(2): 1-12.

11. HO TS, et al. Clinical and laboratory predictive markers for acute dengue infection. J Biomed Sci, 2013; 20(1): 1-8.

12. HUMAYOUN MA, et al. Multiple dengue serotypes and high frequency of dengue hemorrhagic fever at two tertiary care hospitals in Lahore during the 2008 dengue virus outbreak in Punjab, Pakistan. Int J Infect Dis, 2010; 14 (3): 54 59.

13. JAYADAS TTP, et al. The clinical profile, hematological parameters and liver transaminases of dengue NS1 Ag positive patients admitted to Jaffna Teaching Hospital, Sri Lanka. BMC Res Notes, 2019; 12(604): 1-5.

14. KRISHNAMOORTHY S, et al. Hepatitis and thrombocytopenia: markers of dengue mortality. Tropical Doctor, 2017; 47(2): 136-141.

15. LAM PK, et al. The value of daily platelet counts for predicting dengue shock syndrome: Results from a prospective observational study of 2301 Vietnamese children with dengue. PLoS Negl Trop Dis 11, 2017; 4: 1-20.

16. LEE IK, et al. Prognostic Factors in Adult Patients with Dengue: Developing Risk Scoring Models and Emphasizing Factors Associated with Death $\leq 7$ Days after Illness Onset and $\leq 3$ Days after Presentation. J Clin Med, 2018; 7(11): 1-15.

17. OPS/OMS. Dengue: Guías de atención para enfermos en la región de las Américas. 2010. Disponível em: https://www.paho.org/hq/dmdocuments/2012/Guias-atencion-enfermos-Americas-2010-esp.pdf Acessado em: 05 de fevereiro de 2020.

18. PAHO. Dengue: guidelines for patient care in the Region of the Americas. 2016. Disponível em: https://iris.paho.org/bitstream/handle/10665.2/31207/9789275118900-eng.pdf?sequence= 1 \& isAllowed=y Acessado em: 05 de fevereiro de 2020.

19. RALAPANAWA U, et al. Value of peripheral blood count for dengue severity prediction. BMC Res Notes 11, 2018; 400: 1-6.

20. SHIVBALAN S, et al. Predictors of spontaneous bleeding in Dengue. Indian J Pediatr, 2004; 71(1): $33-36$.

21. TAVAKOLIPOOR $P$, et al. Clinical features and laboratory findings of dengue fever in German travellers: A singlecentre, retrospective analysis. Travel Med. Infect. Dis, 2016; 14: 39-44.

22. TEE HP, et al. Risk factors associated with development of dengue haemorrhagic fever or dengue shock syndrome in adults in Hospital Tengku Ampuan Afzan Kuantan. The Medical journal of Malaysia, 2009; 64(4): 316-320.

23. THOMAS L, et al. Predictors of severe manifestations in a cohort of adult dengue patients. J Clin Virol, 2010; 48(2): 96-99.

24. VISHNURAM P, et al. Evaluation of Febrile Thrombocytopenia Cases in a South Indian Tertiary Care Hospital. J Assoc Physicians India, 2018; 66(5): 61-64.

25. WONG JGX, et al. Identifying Adult Dengue Patients at Low Risk for Clinically Significant Bleeding. PLoS ONE 11, 2016; $2: 1-12$.

26. WORLD HEALTH ORGANIZATION. Dengue: guidelines for the diagnosis, treatment, prevention and control. 2009. Disponível em: https://www.who.int/tdr/publications/documents/dengue-diagnosis.pdf. Acessado em: 06 de fevereiro de 2021.

27. YEH C-Y, et al. Symptoms associated with adverse dengue fever prognoses at the time of reporting in the 2015 dengue outbreak in Taiwan. PLoS Negl Trop Dis, 2017; 11(12): 1-15. 